\title{
Propiedades cualitativas de un modelo de inventario con rotura
}

\author{
Luis Lara ${ }^{1}$, Fernando Roda ${ }^{1}$ \\ ${ }^{1}$ FCEIA, Universidad Nacional de Rosario, \\ Avda. Pellegrini 250, (S2000BPT) Rosario, Argentina. \\ lplara@fceia.unr.edu.ar, ferrodamarani@yahoo.com.ar
}

\begin{abstract}
Muchas empresas de producción o comercialización invierten grandes sumas de dinero en la gestión y mantenimiento de sus inventarios. Gran parte de esos costos son ocasionados por la pérdida total o parcial del valor del ítem en especial cuando estos son almacenados durante periodos de tiempo prolongados. En este trabajo se presenta un análisis de la dinámica de un modelo de inventario con rotura de ítems por contacto, considerando la demanda y el lead-time constantes. Si bien el modelo es no lineal, mediante la teoría cualitativa de ecuaciones diferenciales fue posible describir rigurosamente las propiedades generales sin la necesidad de recurrir a simulaciones numéricas. Además se establecen una serie de criterios para la formulación de una política de reposición exitosa y se propone una estrategia de venta que evita llegar a niveles de stock nulo. Por último mediante la simulación son estudiados los efectos del comportamiento estocástico y periódicos de la demanda.
\end{abstract}

Keywords: Sistemas Dinámicos, Inventario con Roturas, Dinámica Industrial.

\section{Introducción}

En la gestión de inventarios, muchas empresas buscan soporte en sistemas informáticos de planificación de recursos (ERP) que para tal fin brindan un conjunto de soluciones generales que, si bien poseen un alto grado de customización, a veces no se ajustan a las características propias de cada negocio. En estos casos se recurre al reajuste de los parámetros del aplicativo o los encargados de compras deben conformarse con disponer de información incompleta. Así es que muchos encargados suelen tomar decisiones basándose en su experiencia o intuición de negocio.

Cuando, por las características del negocio, una compañía debe invertir gran porcentaje de su capital en stock, es vital, para sostener una alta rentabilidad, disponer de herramientas que permitan el estudio de cada escenario particular, de manera de obtener políticas de reposición más confiables. En este artículo se pondrán en práctica algunas de estas herramientas que conforman un componente de importancia en el análisis de sistemas de información que brinden soporte a la gestión de stock. El estudio de la dinámica de sistemas en este tipo de problemas permite analizar las formas en que las políticas de decisión influyen sobre el comportamiento del sistema permitiendo identificar problemas y oportunidades de mejora. En especial, la dinámica indus- 
trial es una herramienta útil para el diseño de sistemas complejos [13] como el que se presenta en este trabajo. En ellos las políticas de decisión y control ocasionan flujos de realimentación de información que pueden ser tratados convenientemente utilizando los conceptos de la dinámica industrial. De esta manera se logra identificar la interacción entre las variables, los flujos y niveles involucrados [1][10].

En la literatura especializada, muchos modelos de inventarios han sido ampliamente estudiados con distintos métodos de investigación de operaciones, siendo los más conocidos aquellos que resuelven problemas de optimización sobre el lote de compra o el periodo abastecimiento (EOQ). Este tipo de modelos asume que los ítems en stock no son afectados por el paso de tiempo y consideran un abastecimiento instantáneo. Sin embargo, este no es siempre el caso, de hecho en muchas empresas industriales, el abastecimiento se produce a tasas de producción continuas. Incluso en las empresas comerciales las órdenes llegan inicialmente a un depósito y puede tomarle días al departamento de recepción la transferencia de estos al almacén. Por lo tanto suele apelarse a otro tipo de representación que permita modelar sistemas con reaprovisionamiento no-instantáneo en el dominio continuo.

Muchas clases de productos que ingresan en los almacenes y se conservan durante una determinada cantidad de tiempo, no suelen estar disponibles en su totalidad al momento de su despacho. A estos productos se los conoce en la literatura como "deteriorating items" y la perdida total o parcial de su valor se debe a diferentes causas como la caducidad de artículos perecederos, el daño físico por roturas o golpes, la obsolencia ante cambios en la tecnología o el mercado, la evaporación de productos químicos, etc. [8-9]

Los problemas de inventarios de los "deteriorating ítems" fueron estudiados por primera vez por Whitin [14] quien analizó como algunos productos "de moda" perdían valor con el paso del tiempo. Siguiendo esta línea, varios trabajos de investigación han sido publicados considerando a la función de deterioro constante o dependiente del tiempo [2-4]. Mandal y Maiti [15][16][5] introdujeron a este tipo de modelos una clase interesante de ítems hechos de materiales como el vidrio, la porcelana o la cerámica en los cuales las características del material y la disposición que adquieren en el almacén hacen que estos productos se rompan fácilmente al entrar en contacto. La función de perdida de esta clase de artículos depende de la cantidad de stock acumulado y esta relación suele involucrar no linealidades muy difíciles de tratar analíticamente. Sin embargo, mediante el estudio cualitativo de las soluciones [11], la simulación numérica y el análisis asintótico [12] se logra describir las propiedades que caracterizan adecuadamente su dinámica.

Este trabajo expone las propiedades de un sistema de gestión de stock considerando la rotura de ítems por contacto y las políticas de control que permitan restablecer el stock al nivel deseado cualquiera sean las condiciones iniciales. Con el objetivo de evitar periodos de existencia nula (con los consecuentes márgenes negativos que esto conlleva) y considerando la restricción que se le plantea al minorista al no poseer información confiable y oportuna del nivel de stock disponible por el proveedor. Es por esto que la política de reposición dependerá solamente del nivel minorista.

El trabajo esta organizado de la siguiente manera: en la sección 2 se desarrolla el modelo de inventario con rotura, la sección 3 comprende un estudio de la estabilidad del sistema definiendo algunas restricciones para la política de reposición. Luego en la sección 4 se propone una estrategia de control de ventas que evita los periodos de 
existencias nulas. La sección 5 extiende el estudio a un caso particular donde la política de reposición es lineal respecto al nivel de stock real. Por último en la sección 6 se muestra un primer acercamiento al caso de demanda variable mediante una simulación numérica del modelo.

\section{Modelo Continuo con Rotura}

El sistema en estudio está compuesto por un comercio minorista que vende productos según una demanda que se considera constante y es abastecido por un único proveedor de acuerdo a las ordenes de compra que el primero va emitiendo según su política de aprovisionamiento. Los artículos recibidos por el minorista pueden romperse por el simple contacto entre uno y otro durante el almacenaje o manipulación dentro del almacén. Una vez hecho el pedido al proveedor, en el proceso productivo (o de adquisición) necesario para abastecer la orden se insume una cantidad de tiempo (o leadtime) correspondiente a todos los procesos administrativos y de gestión necesarios para el abastecimiento.

También podría considerarse que el flujo de mercadería es interno, teniendo entonces un departamento de comercialización abastecido por un departamento de producción o abastecimiento.

Por tanto, el modelo propuesto contiene 2 niveles $x(t)$ : nivel de inventario del minorista o dpto. de comercialización. $y(t)$ : nivel de artículos pendientes o en proceso de producción.

En la Fig. 1 se presenta el cursograma correspondiente.

Los parámetros utilizados son:

$s$ : demanda por unidad de tiempo.

$x_{d}$ : nivel de inventario deseado.

$a$ : proporción de artículos rotos por contacto.

$d$ : demora promedio de producción o compra.

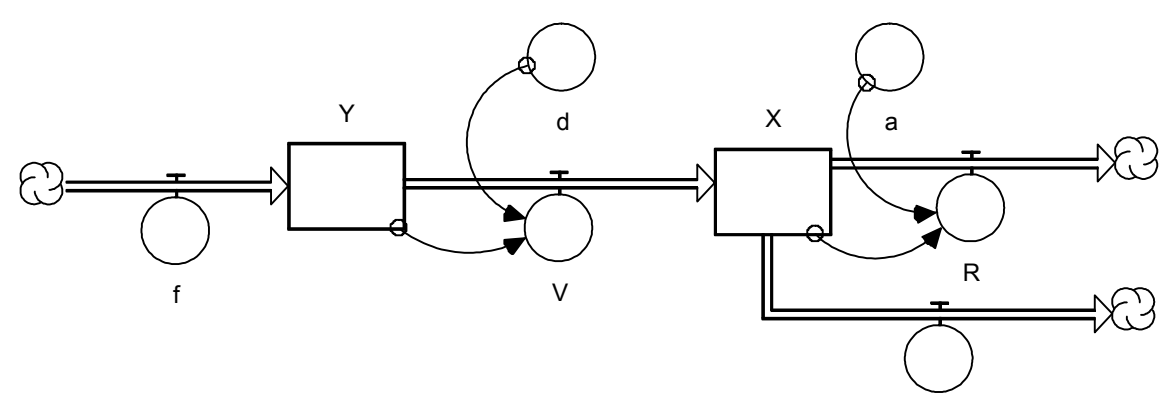

$\mathrm{s}$

Fig. 1. Cursograma del modelo propuesto

$f(x)$ : es siempre positiva y establece la política de pedidos de aprovisionamiento. Al no disponerse de información precisa del nivel de ordenes pendientes en el proveedor, 
esta política es representada como una función que solo depende del nivel de inventario minorista.

La tasa de pérdida de mercadería por rotura [7] es $R=a x^{2}, a>0$.Teniendo en cuenta la demora en la gestión del pedido, en el proceso de producción y envío de la mercadería, los artículos pedidos ingresan al almacén minorista con una tasa $V=y / d$.

El sistema de ecuaciones resultante es

$$
\begin{gathered}
\dot{x}=-s+\frac{y}{d}-a x^{2}, \\
\dot{y}=-\frac{y}{d}+f(x) .
\end{gathered}
$$

Las ecuaciones son invariantes frente al grupo de transformación:

$$
t \leftarrow \frac{t}{d}, a \leftarrow s d^{2} a, x \leftarrow \frac{x}{s d}, y \leftarrow \frac{y}{s d}, f \leftarrow \frac{f}{s},
$$

el sistema se rescribe como

$$
\begin{aligned}
& \dot{x}=y-1-a x^{2}, \\
& \dot{y}=-y+f(x) .
\end{aligned}
$$

Solo se pondrá atención en las soluciones de $x, y \geq 0$.

\section{Propiedades cualitativas}

\section{Puntos fijos y estabilidad}

Los puntos fijos $\left(x^{*}, y^{*}\right)$ de la Ec. (2) son aquellos que satisfacen las ecuaciones $y^{*}=1$ $+a\left(x^{*}\right)^{2}, y^{*}=f\left(x^{*}\right)$. Pero, siendo el objetivo la estabilización del nivel de inventario $x$ en el valor deseado $x_{d}$, la primera condición que se impondrá al modelo es la existencia de un único punto fijo positivo ubicado en $\left(x_{d}, y_{d}=1+a x_{d}^{2}\right)$. Además, la política $f(x)$ deberá ser tal que tenga a $x_{d}$ como raíz positiva de la ecuación:

$$
1+a x^{* 2}=f\left(x^{*}\right) \text {. }
$$

La estabilidad del punto fijo la determinamos linealizando las Ecs.(2) alrededor de este [11]. La matriz Jacobiana en el punto fijo es

$$
J=\left(\begin{array}{cc}
-2 a x^{*} & 1 \\
f^{\prime}\left(x^{*}\right) & -1
\end{array}\right),
$$

los autovalores de la matriz $J$, se escriben como 


$$
\lambda_{ \pm}=\frac{1}{2} \tau\left(1 \pm \sqrt{1-4 \frac{\Delta}{\tau^{2}}}\right)
$$

siendo

$$
\begin{gathered}
\tau=-\left(1+2 a x^{*}\right), \\
\Delta=2 a x^{*}-f^{\prime}\left(x^{*}\right),
\end{gathered}
$$

Dado que estamos interesados en que el punto fijo $\left(x_{d}, y_{d}\right)$ sea estable, la parte real de los autovalores debe ser negativa, y siendo $\tau<0$, es necesario que $\Delta>0$. Por lo tanto debe cumplirse que

$$
f^{\prime}\left(x_{d}\right)<2 a x_{d} .
$$

Además, como la traza es siempre negativa no existen soluciones periódicas en el entorno del punto fijo.

Cumplimiento de la inecuación (8) depende del diseño de la política de reposición, del nivel de inventario deseado y del valor que asuma el índice de rotura. Si bien a este último se lo ha considerado como un parámetro constante y conocido, en el sistema real presentará fluctuaciones difíciles de estimar. Por otro lado, y a pesar de que pudiera garantizarse el cumplimiento de (8), la dinámica del sistema no será la misma y dependerá de la forma en que los parámetros definen los valores de $\Delta$ y $\tau$. Así, de acuerdo a la posición que estos tengan en el plano $\Delta \tau$, el punto fijo puede ser una espiral, un nodo estable, una estrella o un nodo degenerado y en consecuencia esto determinará el grado de amortiguamiento, la velocidad de respuesta y la frecuencia de oscilación. De hecho, si $\tau^{2}-4 \Delta>0$, la dinámica de la respuesta en el tiempo será sobreamortiguada, pero si en cambio $\tau^{2}-4 \Delta>0, \lambda$ resulta complejo y $x(t) \mathrm{e}$ $y(t)$ presentarán oscilaciones. Remplazando (6) y (7) en esta última inecuación se obtiene una importante propiedad, ya que si la política de reposición es diseñada de modo tal que $f^{\prime}(x)>0$ se logra evitar las oscilaciones en los niveles de stock en las proximidades del punto fijo, independientemente del valor que tome $a$.

\section{Nullclines}

Para determinar las propiedades cualitativas de las trayectorias en el plano de fases $(x, y)$, el flujo asociado a la Ec. (2) es: $\vec{F}:=\left(y-1-a x^{2},-y+f(x)\right)$.

Se define la isoclina nula $\gamma_{1}$ como la curva en la cual $F_{x}=0$, esto es $y=1+a x^{2}$. Análogamente, la isoclina nula $\gamma_{2}$ esta definida por la curva $y=f(x)$ en donde $F_{y}=0$. Entonces, de acuerdo a la restricción (8), en el punto de intersección de ambas curvas $\left(x_{d}, y_{d}\right)$, la pendiente de $\gamma_{1}$ debe ser mayor a la pendiente de 
$\gamma_{2}$. De esta manera quedan determinadas cuatro regiones en el espacio de fases como se muestra en la Fig.2. Independientemente de la definición que tome $f(x)$, el flujo tiene componente horizontal positiva por encima de $\gamma_{1}$ y negativa bajo ella, ya que $F_{x}>0$ si y solo si $y>1+a x^{2}$. Por otro lado, la componente vertical es negativa sobre la curva $\gamma_{2}$ y positiva bajo ella ya que $F_{v}<0$ si y solo si $y>f(x)$.

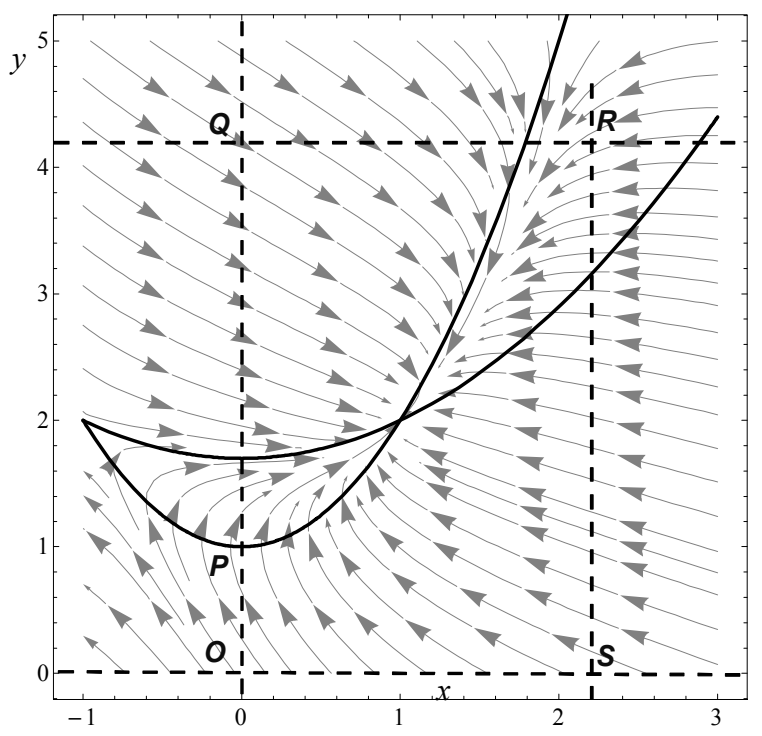

Fig. 2. Plano de fase y región de atracción $\Omega$ para un caso particular.

Para demostrar la convergencia del flujo hacia el valor deseado $x_{d}$,se define una región cerrada en el plano de fase que contiene a $\left(x^{*}, y^{*}\right)$, de modo tal que las trayectorias que ingresan a ella se mantienen allí o eventualmente pueden salir a través de un rango de valores de $y \quad(x=0)$. La región $\Omega$ queda definida por un rectángulo de vértices $O Q R S$ como se muestra en la Fig. 2, donde $O=(0,0), Q=\left(x_{1}, 0\right)$, $R=\left(x_{1}, y_{1}\right), S=\left(0, y_{1}\right)$, con $x_{1}$ e $y_{1}$ arbitrarios tales que:

$$
\begin{gathered}
x_{1}>x^{*}, \\
y_{1}<1+a x_{1}^{2}, \\
y_{1}>f(x) \text { para } 0 \leq x \leq x_{1} .
\end{gathered}
$$


El comportamiento de las trayectorias en las fronteras de la región es el siguiente:

1. Sobre el segmento $\overline{S R}, y<y_{1}$ y dado que hemos impuesto la restricción (10) la componente horizontal del flujo es $F_{x}=y-1-a x_{1}^{2}<0$.

2. Sobre el segmento $\overline{Q R}$, de acuerdo a (11) $F_{y}=-y_{1}+f(x)<0$.

3. Sobre $\overline{O S}$ y en todo el eje $y=0$ la componente $F_{y}$ es siempre positiva ya que independientemente de la política de reposición utilizada, $f$ es positivo.

4. Sobre el segmento $\overline{O Q}$ vemos que la isoclina $\gamma_{1}$ intercepta al eje $x=0$ en $y=1$; por lo tanto cualquiera sea $f>0$, sobre el eje $y$ tenemos $F_{x} \geq 0$ para $y \geq 1$ y $F_{x}<0$ si $y<1$ lo que representa una desventaja en la dinámica ya que las trayectorias pueden escapar de la región hacia niveles de inventario negativos.

Teniendo en cuenta las propiedades $1,2,3$ y 4 toda trayectoria que ingresa a la región permanecerá ella o puede retirarse hacia $x<0$ a través de la ventana $\overline{O P}$ en donde $y<1$. Esto indica que si los niveles de productos pendientes del proveedor (o en proceso de elaboración) son memores a la demanda esperada durante el lead-time medio, entonces el minorista podrá quedarse sin mercadería.

\section{Estrategia de comercialización}

En la sección anterior se determinó la existencia de condiciones iniciales que conducirán a valores negativos de inventario, y esto presenta dos claras desventajas: 1) por un lado, los clientes que soliciten mercadería en este periodo de existencia nula, no podrán satisfacer su demanda en ninguna medida y están obligados a buscar otros proveedores o, en el mejor de los casos, a esperar hasta haberse restablecido los niveles de stock. Es evidente que bajo este escenario, la cantidad de clientes irá disminuyendo. Si esto sucede, puede desencadenarse un periodo de subocupación de capacidad instalada con un claro perjuicio en los márgenes de ganancia. 2) por otro lado, al paralizarse las ventas, el minorista (o dpto. de comercialización) debe enfrentar períodos de costos fijos, pero sin ingresos por ventas.

Para evitar esta dificultad, se propone una estrategia de venta que permite controlar la demanda, cuando los niveles de stock son suficientemente bajos. Una forma de lograrlo es mediante el uso de restricciones en las cantidades vendidas aplicando cotas superiores variables. Esta estrategia es modelada redefiniendo la tasa de salida del nivel $x$ respecto de la demanda $s$ como un producto de funciones de la forma $h(x) \times s \rightarrow s(x)$, donde $s$ es la tasa de artículos requeridos por los clientes, definida en la Sec. 2, y que en nuestro estudio consideramos constante. La función $h(x)$ modela la estrategia de venta que debe cumplir las siguientes condiciones:

$$
\begin{gathered}
h(0)=0, \\
h(x)=1 \quad \text { para } x \geq \varepsilon>0 . \\
0<h(x)<1 \text { y } h^{\prime}(x) \geq 0 \text { para } 0<x<\varepsilon,
\end{gathered}
$$


donde $\varepsilon<x_{d}$ y su valor dependerá de la intensidad o rigidez de la estrategia de comercialización, siendo que un mayor valor $\varepsilon$ implica una mayor previsión de la escasez de stock y por consiguiente el crecimiento de las cotas de ventas a aplicar será más lento. Teniendo en cuenta la transformación de escala, las Ecs. (2) se rescriben como

$$
\begin{gathered}
\dot{x}=y-h(x)-a x^{2}, \\
\dot{y}=-y+f(x) .
\end{gathered}
$$

Entonces para $x>\varepsilon$ la nueva dinámica tiene las mismas propiedades que el modelo original y el flujo en $\Omega$ permanece sin cambios para estos valores de $x$. Pero sobre el segmento $\overline{O Q}$, donde $x=0$, la dinámica cambia favorablemente. Dado que la componente horizontal del flujo es $F_{x}=y-h(x)-a x^{2}$ y como $h(x)=0$ para $x=0$, sobre dicho segmento las trayectorias solo pueden ingresar a la región. La Fig. 3 muestra un ejemplo del plano de fase resultante donde puede observarse como las orbitas ingresan y quedan confinadas en la región $\Omega$. Además, como dicha región puede establecerse arbitrariamente grande, cumpliéndose las restricciones (12), cualquier condición inicial positiva conducirá a una orbita que quedará atrapada por $\Omega \mathrm{y}$ por lo tanto $x$ e $y$ resultarán acotados.

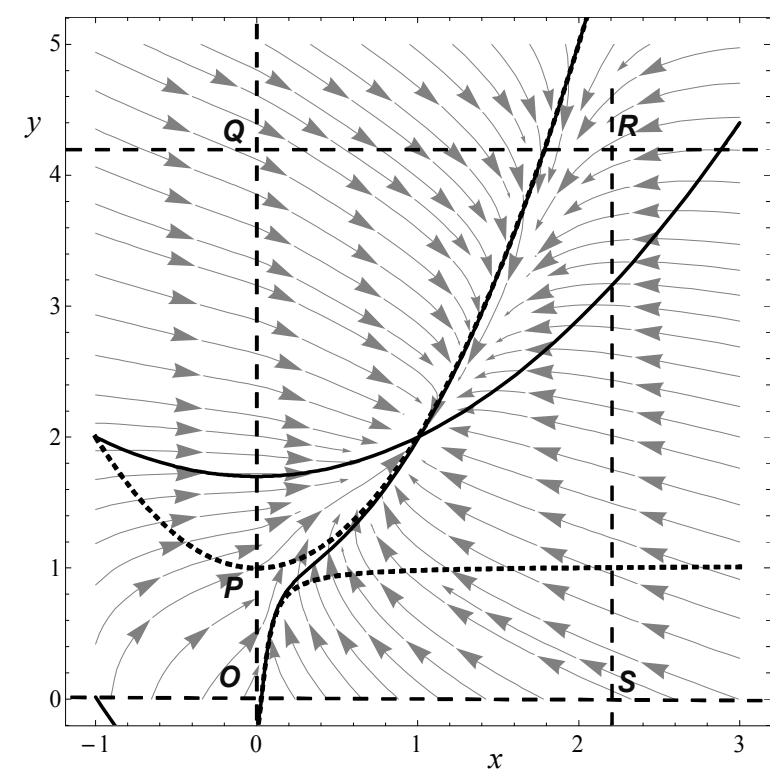

Fig. 3. Efecto de la estrategia de comercialización en las orbitas del sistema.

Sin embrago, lo dicho no garantiza que la solución tienda al valor deseado $\left(x_{d}, y_{d}\right)$ ya que esta podría converger a una solución periódica. Para demostrar que la dinámi- 
ca es no periódica desarrollamos un análisis asintótico [6],[12]. Primero integramos la Ec.(13b) resultando

$$
y(t)=\frac{y_{0}+\int_{0}^{t} e^{t^{\prime}} f\left(x\left(t^{\prime}\right)\right) d t^{\prime}}{e^{t}}
$$

y aplicando la regla de L'Hopital obtenemos

$$
\lim _{t \rightarrow \infty} y=\lim _{t \rightarrow \infty} f(x),
$$

luego, estando $y$ acotada, el limite es finito. Asintóticamente la Ec.(13a) es $\dot{x} \cong f(x)-h(x)-a x^{2}$ y por ser esta una ecuación de primer orden en la recta no admite soluciones oscilatorias. Entonces dado que $x$ está acotada resulta $x \rightarrow$ constante cuando $t \rightarrow \infty$. Además, de acuerdo a (14) $y \rightarrow$ constante por ser $f$ univaluada. Por lo tanto, dado que existe un único punto fijo en $\Omega$, tenemos

$$
\begin{aligned}
& \lim _{t \rightarrow \infty} x=x_{d}, \\
& \lim _{t \rightarrow \infty} y=y_{d},
\end{aligned}
$$

Así, todas las orbitas que ingresan a $\Omega$ asintóticamente convergen al valor deseado y las soluciones periódicas no son posibles. Otra manera de llegar al mismo resultado es a través del criterio de Dulac's [11] ya que en la región $\Omega$ la divergencia del flujo es de signo constante y por lo tanto quedan excluidas las soluciones periódicas en dicha región.

\section{Política Lineal}

Se propone ahora considerar una política de reposición lineal respecto al nivel de stock minorista $x$. Esta es:

$$
f(x)=\alpha+\beta x,
$$

donde los parámetros $\alpha$ y $\beta$ deberán fijarse con el objeto de brindar las mejores características posibles en el control del inventario. Luego el sistema adimensional (2) se rescribe como:

$$
\begin{aligned}
& \dot{x}=y-1-a x^{2}, \\
& \dot{y}=-y+\alpha+\beta x .
\end{aligned}
$$

Teniendo en cuenta la Ec. (3) obtenemos

$$
x_{ \pm}^{*}=\frac{\beta}{2 a}\left(1 \pm \sqrt{1+\frac{4 a(\alpha-1)}{\beta}}\right),
$$


y debido a que buscamos que $x^{*}=x_{d}$ sea el único punto fijo positivo, resulta que $\alpha>1$. Fijando los valores de $x_{d}, a, \alpha$ y usando la Ec. (3) el valor de $\beta$ queda determinado como

$$
\beta=\frac{1+a x_{d}^{2}-\alpha}{x_{d}},
$$

Conforme a la restricción impuesta por Ec.(8), $\beta$ debe ser tal que se cumpla $\beta<2 a x_{d}$ o remplazando en (20), $\alpha>1-a x_{d}^{2}$. Sin embargo, como impusimos que $\alpha>1$, esta condición siempre se cumple y podemos concluir que el parámetro $\alpha$ es el único que determinará la estabilidad del punto fijo positivo $\left(x_{d}, y_{d}\right)$, cualquiera sean los valores de $a$ y $x_{d}$.

La Fig 4 muestra el espacio de las fases del sistema (18) donde se observa la existencia de una zona, sobre el eje $x=0$, en la cual las trayectorias escapan hacia niveles negativos de inventario.

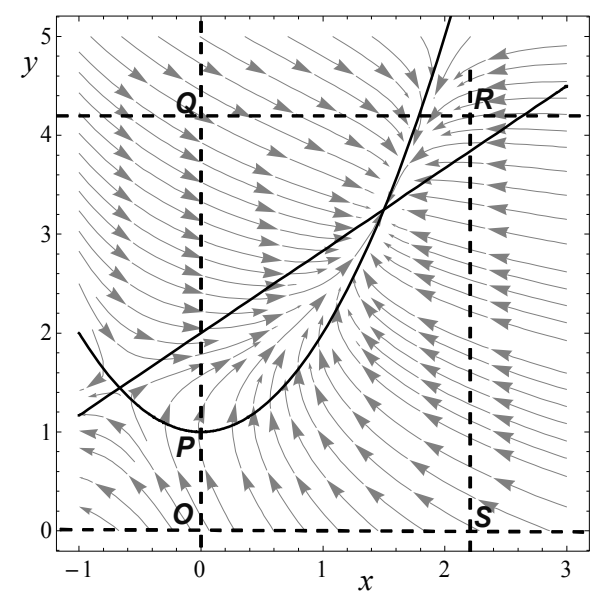

(a)

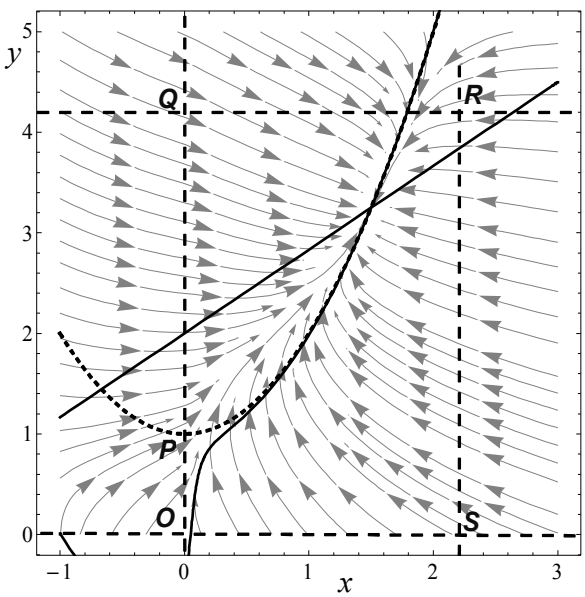

(b)

Fig. 4. Plano de fases del sistema utilizando una política de reposición lineal. a) Sin aplicar cotas de venta b) Con la aplicación de cotas de venta.

Aplicando la estrategia de venta $h(x)$ presentada en la Sec. 4, se cancela el flujo hacia regiones negativas de inventario tal como se muestra en la Fig. 4 


\section{Demanda no Estacionaria}

Hasta ahora, y a los fines del modelo propuesto, se representó a la demanda como una función constante. Sin embargo, esta simplificación a veces no es adecuada al analizar el sistema real. La demanda constituye una verdadera variable aleatoria dependiente del tiempo cuya distribución no siempre es conocida. Si dicha distribución se conoce o puede aproximarse estadísticamente, un análisis numérico puede arrojar información relevante sobre el comportamiento del inventario.

A continuación se presentan dos casos particulares para modelar el comportamiento de la demanda. En el sistema propuesto en la Sec. 4,

$$
\begin{gathered}
\dot{x}=y-h(x) s(t)-a x^{2}, \\
\dot{y}=-y+f(x) .
\end{gathered}
$$

se considerará a $s(t)$ como una función periódica o aleatoria. Dado que en cualquiera de los dos casos $s(t)$ puede considerarse acotada, es posible reproducir los resultados de la Sec. 3.2 para demostrar la existencia de la zona de atracción $\Omega$, razón por la cual no son incluidos en este trabajo

\section{Demanda periódica}

Para cierta clase de artículos de estación la demanda puede ser representada mediante $s(t)=1+A \operatorname{sen}(\omega t)$. Hemos realizado numerosos experimentos numéricos tomando los valores iniciales en la zona de atracción. Sin bien la dinámica es no lineal, los experimentos no mostraron indicios de soluciones caóticas. Asintóticamente las orbitas convergen a una curva periódica que solo depende de los parámetros del sistema, y siempre está ubicada alrededor del punto fijo perteneciente al sistema de demanda estacionaria. Esta propiedad es común en los sistemas no lineales sometidos a una perturbación armónica [17]

En la Fig. 5 se muestra los resultados numéricos obtenidos mediante el método de Runge Kutta de cuarto orden tomado $A=0,2 ; w=1,68 ; a=0,01$; la política: $f(t)=25,64-3 x(t)$ para $x<8,55, f(t)=0$ para $x \geq 8,55$, y la condición inicial $x_{0}=9 ; y_{0}=3$. Se puede observar claramente cómo las trayectorias del caso periódico se aproximan a un ciclo alrededor del punto fijo obteniéndose una respuesta oscilatoria no amortiguada.

A modo de ejemplo, en la Fig. 6 se presentan las orbitas del caso periódico para la condición inicial anterior (línea sólida) y una nueva condición $x_{0}=7, y_{0}=1$ (línea entrecortada). Estas trayectorias, contenidas en la zona de atracción, convergen a la misma solución periódica. 

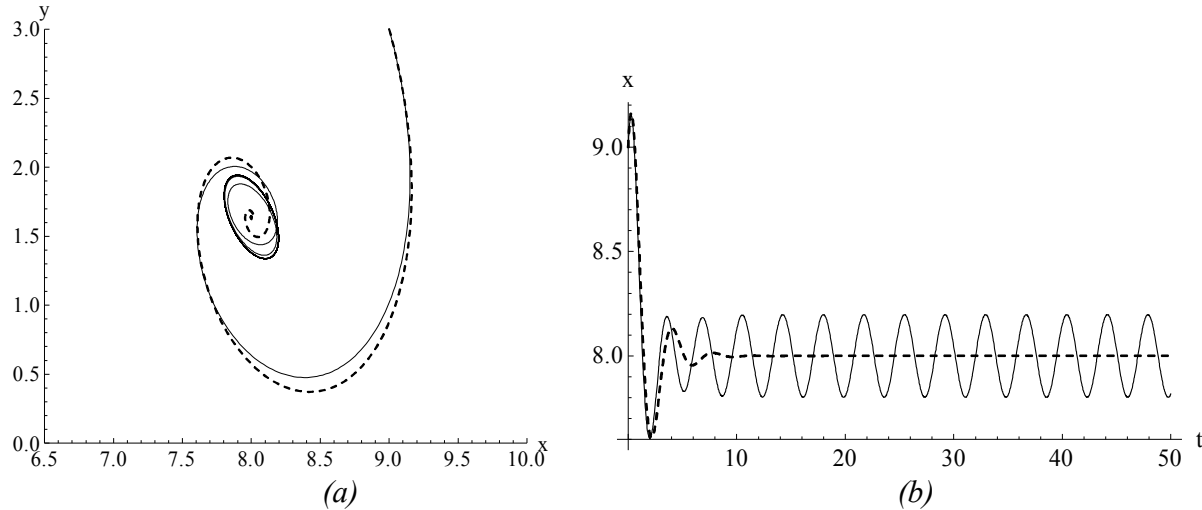

(b)

Fig. 5. Resultados de los experimentos numéricos para una demanda constante (línea entrecortada) y a una demanda periódica (línea sólida). a) Trayectorias en el plano de fases. b) Evolución del nivel minorista $x$.

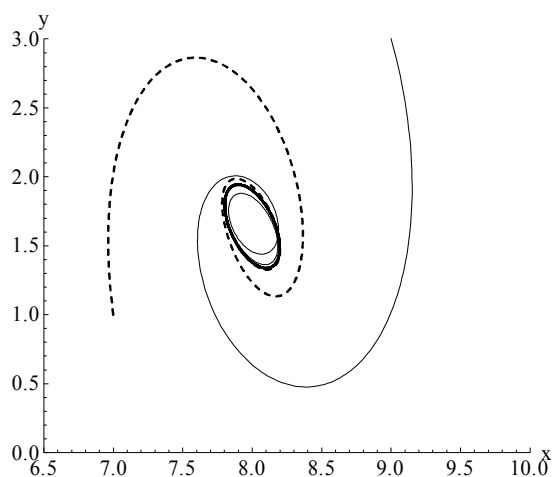

(a)

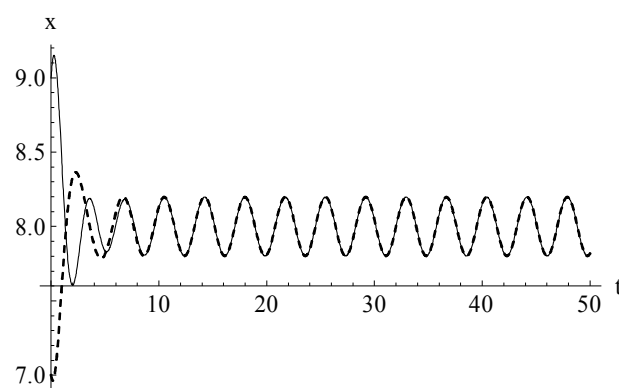

(b)

Fig. 6. Resultados numéricos para el caso periódico considerando las condiciones iniciales $x_{0}=$ 9 , $y_{0}=3$ (línea sólida) y $x_{0}=7, y_{0}=1$ (línea entrecortada). a) Trayectorias en el plano de fases. b) Evolución del nivel minorista $x$.

En la Fig. 7 se muestra la evolución de las orbitas en el espacio $(x, y, t)$. El tiempo es reescaleado al intervalo $(0,2 \pi / \omega)$. Se puede ver como las orbitas en cada escala de tiempo se van agrupando con el incremento de $t$, hasta formar una sola curva que corresponde a la solución periódica. 


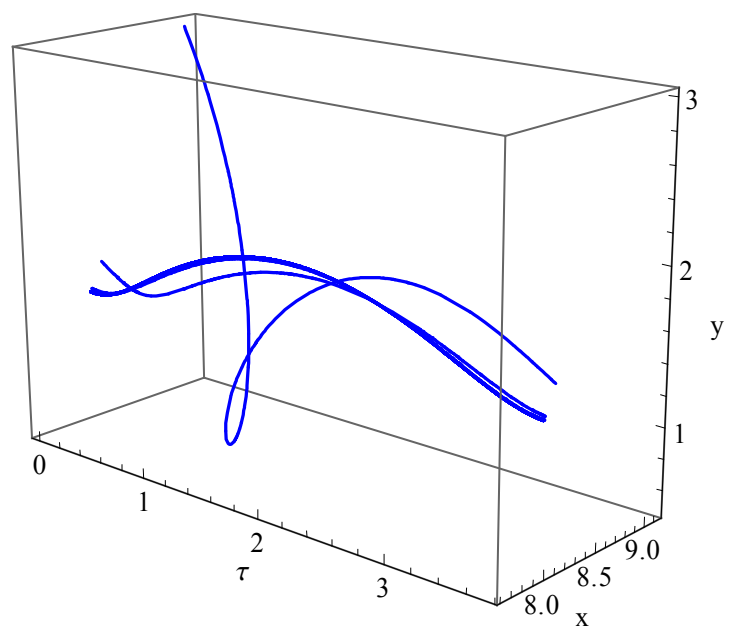

Fig. 7. Orbitas del sistema con demanda periódica en el espacio $(x, y, t)$ reescaleando el tiempo al intervalo del período $(0,2 \pi / \omega)$

\section{Demanda estocástica}

El comportamiento de la demanda en el tiempo siempre contiene componentes estocásticas. En particular cuando las observaciones no evidencian un patrón temporal determinístico, la misma deberá ser modelada mediante una función aleatoria

Como ejemplo consideramos una demanda estocástica cuya distribución es independiente del tiempo como la siguiente: $s(t) \sim N(1,1 / 15)$. Para cada réplica del experimento se determinó una función aleatoria continua que modela la demanda y se integró numéricamente el sistema utilizando los mismos parámetros que en la sección anterior. El experimento aleatorio, constituido por cincuenta replicas, permitió obtener las frecuencias relativas de cada área $(\Delta x, \Delta y)$ del plano de fase, para así determinar el histograma de las variables aleatorias $x(t)$ e $y(t)$. En la Fig. 8 se muestra el resultado obtenido para un determinado valor inicial $\left(x_{0}, y_{0}\right)$. 


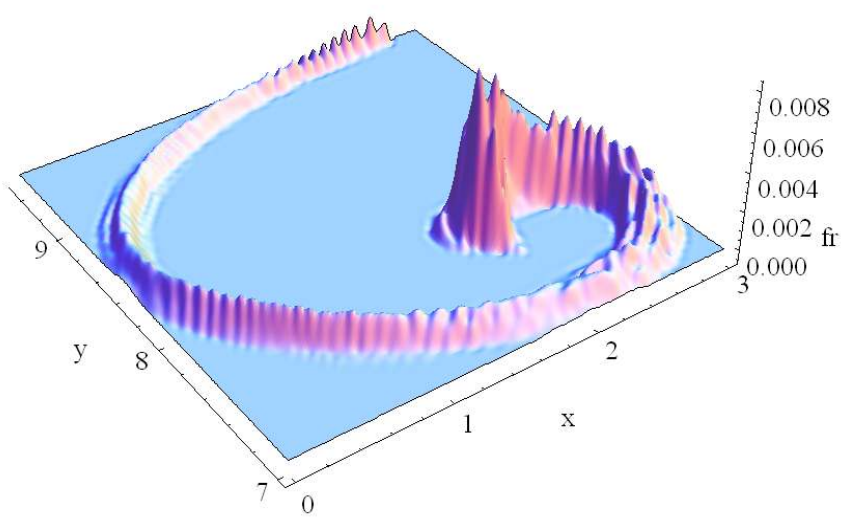

Fig. 8. Histograma de las variables aleatorias $(x(t), y(t))$ con $t$ variando de 0 a 5 , para una condición inicial dada.

En los diferentes experimentos numéricos realizados, se comprobó que cualitativamente las distribuciones de las variables $x(t)$ e $y(t)$ están centradas en los valores del caso determinístico (donde $s(t)=1$ ); y además cuando $t \rightarrow \infty, \overline{x(t)} \rightarrow x_{d} \mathrm{e}$ $\overline{y(t)} \rightarrow y_{d}$.

\section{Conclusiones}

El objetivo fundamental de este trabajo ha sido remarcar la importancia de la teoría cualitativa de ecuaciones diferenciales para analizar rigurosamente las propiedades correspondientes a modelos de dinámica industrial. Estas técnicas minimizan el uso de simulaciones numéricas permitiendo obtener resultados más generales. En particular se ha puesto énfasis en un modelo de inventarios con roturas de artículos por contacto obteniendo los siguientes resultados:

- Se determinaron analíticamente las propiedades del sistema.

- Se estableció un criterio que permite diseñar de una política de reposición que logra estabilizar los niveles al valor deseado, trabajando incluso con la restricción de no disponer de información sobre los niveles del proveedor. Sin embargo no se incluyeron en el modelo los costos involucrados con los cuales podría establecerse con mejor criterio los parámetros de la política.

- Se propuso una estrategia de comercialización que evita la escasez de stock y mediante una adecuada elección de la política de reposición el sistema es siempre estable, ya que cualquiera sea la condición inicial, la solución evoluciona hacia el valor deseado. 
- A pesar de que las roturas introducen una no linealidad al modelo, el sistema es controlable por medio de un control lineal.

- La incorporación de demandas no estacionarias hace que las ecuaciones no lineales sean no autónomas y como es usual se debió recurrir a la simulación. Entre los casos experimentados, no hemos encontrado soluciones caóticas siendo el comportamiento asintótico oscilante alrededor del punto fijo del sistema autónomo.

Si bien en este modelo no se incluyeron los costos involucrados ya que como primera etapa del trabajo

\section{Referencias}

1. Sterman, J.: Business dynamics: system thinking and modeling for a complex word, Irwin Mc. Graw-Hill, Boston (2000).

2. Saleh, M. et al: A Comprehensive analytical approach for policy analysis of system dynamics models, European Journal of Operational Research 203, pp. 673-683 (2010).

3. Cheng, M., Wang, G.: A note on the inventory model for deteriorating items with trapezoidal type demand rate, Computers \& Industrial Engineering 56, 1296-1300 (2009).

4. Warburton, R.: An exact analytical solution to the production inventory control problem, Int. J. Production Economics 92, 81-96 (2004).

5. Saha al: Inventory models for breakable items with stock dependent demand and imprecise constraints, Mathematical and Computer Modeling (2010), doi:10.1016/j.mcn 2010.07.004.

6. Huang, T.: Asymptotic Analysis of Industrial Dynamical Model, Mathematical and Computer Modelling 36, 1459-1473 (2002).

7. Bhattacharya, D. K.: On multi-item inventory, European Journal of Operational Research 162, 786-791 (2005).

8. Ruxian, L., Hongie, L.: A review on deteriorating inventory study, Journal of Service Science and Management (2010).

9. Goyal, S., Giri, B.: Recent Trends in modeling of deteriorating Inventory, European Journal of Operational Research 134, 1-16 (2010).

10. Forrester, J.: Industrial Dynamics Productivity, Press (1961).

11. Strogatz, S.: Nonlinear Dynamics and Chaos, Westview Press, Cambridge (1994).

12. Roscoe, B.W.: Asymptotic Analysis of differential Equations, Imperial College Press (2006)

13. Prigogine, I.: Exploring complexity, European Journal of Operational Research 30, 97-103 (1987).

14. Whitin, T.M.: Theory of Inventory Management, Princeton University Press, Princeton NJ, (1957).

15. Mandal, M., Maiti, M.: Inventory model for damageable items with stock dependents demand and shortages, Opsearch 34 (3), 155-166 (1997)

16. Mandal, M., Maiti, M.: Inventory of damageable items with variable replenishment rate, stock dependent demand and some units in hand, Applied Mathematic Modeling 23, 799807 (1999)

17. Thompson, J.M.T., Stewart, H.B.: Nonlinear Dynamics and Chaos, John Wiley and Sons, New York (1986). 